\title{
Endoplasmic Reticulum Stress-Related Factors Protect against Diabetic Retinopathy
}

\author{
Wei-Kun Hu, Rong Liu, Han Pei, and Bin Li \\ Department of Ophthalmology, Tongji Hospital, Tongji Medical College, Huazhong University of Science and Technology, \\ Wuhan 430030, Hubei Province, China \\ Correspondence should be addressed to Bin Li, scotopsin22@yahoo.com.cn
}

Received 26 May 2011; Revised 20 August 2011; Accepted 21 September 2011

Academic Editor: Rangasamy Sampathkumar

Copyright ( 2012 Wei-Kun Hu et al. This is an open access article distributed under the Creative Commons Attribution License, which permits unrestricted use, distribution, and reproduction in any medium, provided the original work is properly cited.

The endoplasmic reticulum (ER) is a principal mediator of signal transduction in the cell, and disruption of its normal function (a mechanism known as ER stress) has been associated with the pathogenesis of several diseases. ER stress has been demonstrated to contribute to onset and progression of diabetic retinopathy (DR) by induction of multiple inflammatory signaling pathways. Recent studies have begun to describe the gene expression profile of ER stress-related genes in DR; moreover, genes that play a protective role against DR have been identified. $\mathrm{P} 58^{\mathrm{IPK}}$ was determined to be able to reduce retinal vascular leakage under high glucose conditions, thus protecting retinal cells. It has also been found by our lab that ER-associated protein degradation factors exhibit significantly different expression patterns in rat retinas under sustained high glucose conditions. Future research based upon these collective genomic findings will contribute to our overall understanding of DR pathogenesis as well as identify potential therapeutic targets.

\section{Introduction}

Diabetic retinopathy (DR) is one of the most common complications of diabetes mellitus. The surge in diabetes across the globe has led to DR becoming one of the main causes of blindness. Early clinical manifestations of DR include apoptosis of retinal pericytes and increase in permeability of ocular blood vessels. As a consequence, the protective blood-retinal barrier is broken down, which further results in microaneurysm, hard exudates, retinal edema, and minor bleeding. As the disease progresses, the retinal capillary structure becomes so perturbed that all normally perfusable areas are eventually lost (Figure 1). Collectively, these initial clinical changes are diagnosed as nonproliferative (NP) DR, and the disease is considered to have advanced to a proliferative stage (PDR) once revascularization of the affected region has commenced. At this point, successive clinical observations include retinal neovascular proliferation, vitreous hemorrhage, retinal detachment, and loss of vision. Ultimately, the patient succumbs to blindness. Interestingly, retinal edema progression to involve the macula, the central area of the retina, is considered the principal underlying cause of impaired vision.
Many large-population, multicenter clinical studies have been performed to identify the most significant risk factors of DR onset and progression. It appears that the features of diabetes duration, severity of hyperglycaemic episodes, and elevated blood pressure are directly related to DR [1-4]. Many DR pathoincidence theories have been proposed on the basis of these findings, but none have yet to result in an effective "gold-standard" prophylactic or therapeutic strategy. At present, the preferred clinical treatment process is careful maintenance of blood glucose levels and of blood pressure. Events of neovascularization and capillary nonperfusion are commonly treated by partial retinal photocoagulation and pan-retinal photocoagulation, and macular edema is treated by laser and vitrectomy. At the latest stages of DR, retinal reattachment surgery is available.

Despite a vast amount of investigative effort, the pathoincidence of DR is not completely understood. Research findings have implicated roles for the polyol pathway (aldose reductase-mediated conversion of glucose to sorbitol) [5, 6], protein kinase C (a known mediator of glucose transport) $[7,8]$, advanced glycation end products (AGEs, forming from accumulated glucose), and oxidative stress 


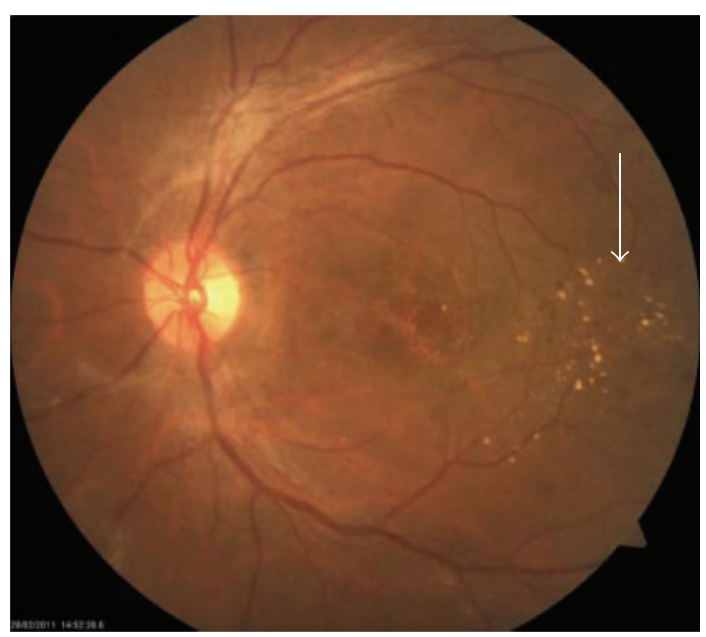

(a)

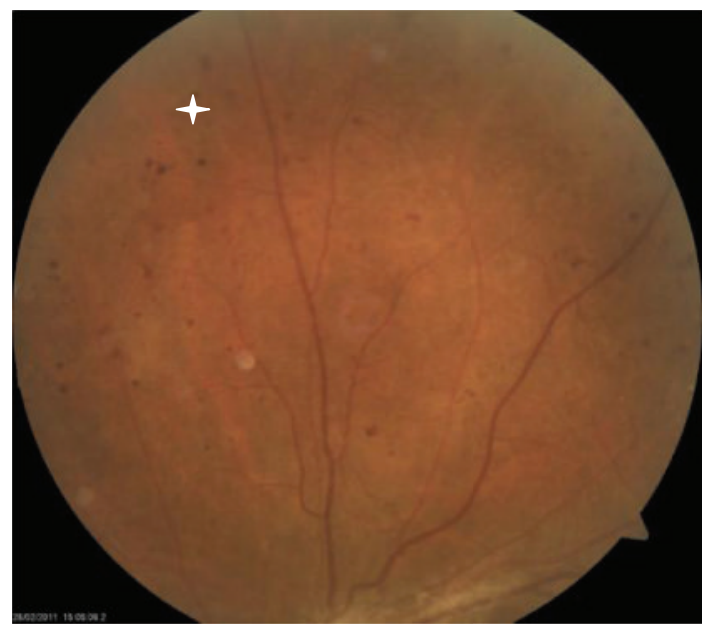

(c)

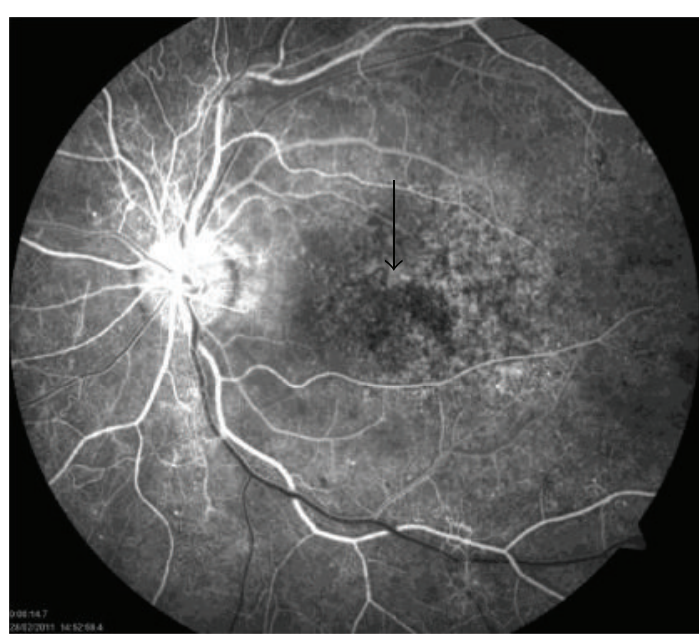

(b)

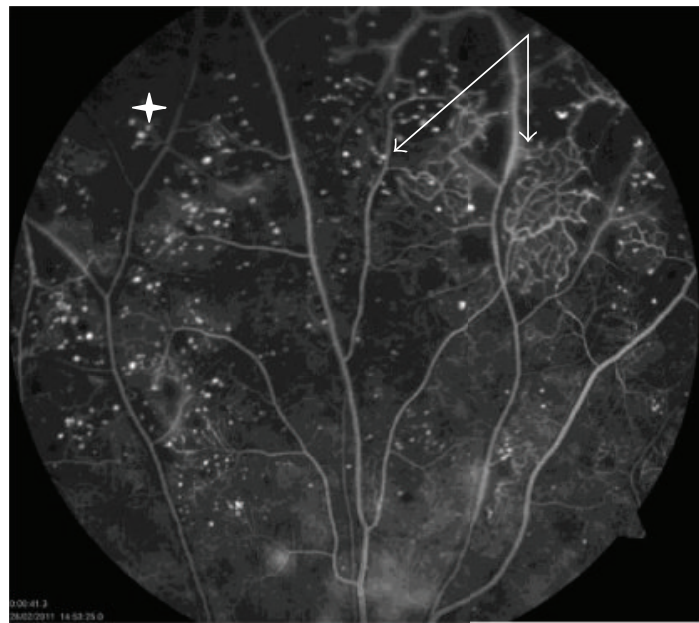

(d)

FIGURE 1: Fundus photographs ( $a, c)$ and retinal fluorescence angiography $(b, d)$ of a patient with proliferative diabetic retinopathy. (a, b) and (c, d) are images of the same part of the retina. The Fundus photographs revealed widely scattered spots on the retina, which represent microaneurysms (white star in c). In addition, we observed that the macular foveal reflex had disappeared. Yellow-white exudates were apparent on the temporal area of the macula (white arrow in a). The angiograms were obtained during the arterial phase (b) and the late arteriovenous phase (d), after injection of dye into an antecubital vein. Retinal neovascularization was observed adjacent to areas of vascular nonperfusion (white arrow in d). The multiple, tiny fluorescent dots (white star in d) are microaneurysms. The blood-retinal barrier breakdown manifests as neovascular lesions, which fluoresce brightly and appear blurred as the dye leaks from the vascular lumina (black arrow in b).

(a by-product of glucose metabolism) [9, 10]. Moreover, specific immune/inflammatory factors and angioincidence factors $[11,12]$ have been implicated in the incidence and development of DR [13-18].

The incidence of DR has also gained the interest of clinical geneticists interested in determining whether heredity may significantly contribute to DR risk. Indeed, many DRsusceptibility genes have been identified by their efforts [1921]. Therefore, DR is believed to have a genetic component, and further research into this mechanism will advance our overall understanding of DR pathogenesis and help to identify targets for potential genome-based therapy.

\section{ER Stress and DR}

Recently, studies into the underlying molecular mechanisms of DR have suggested that endoplasmic reticulum (ER) stress may play important roles in triggering and maintaining the disease state. The ER organelle mediates processing of newly translated proteins, from synthesis and modification to transport. ER stress is the process of the ER adjusting its function, accelerating or decelerating internal machinery to effectively meet the precise needs of the cell under dynamic conditions. For example, the cellular unfolded protein response (UPR) acts to reduce overall protein 


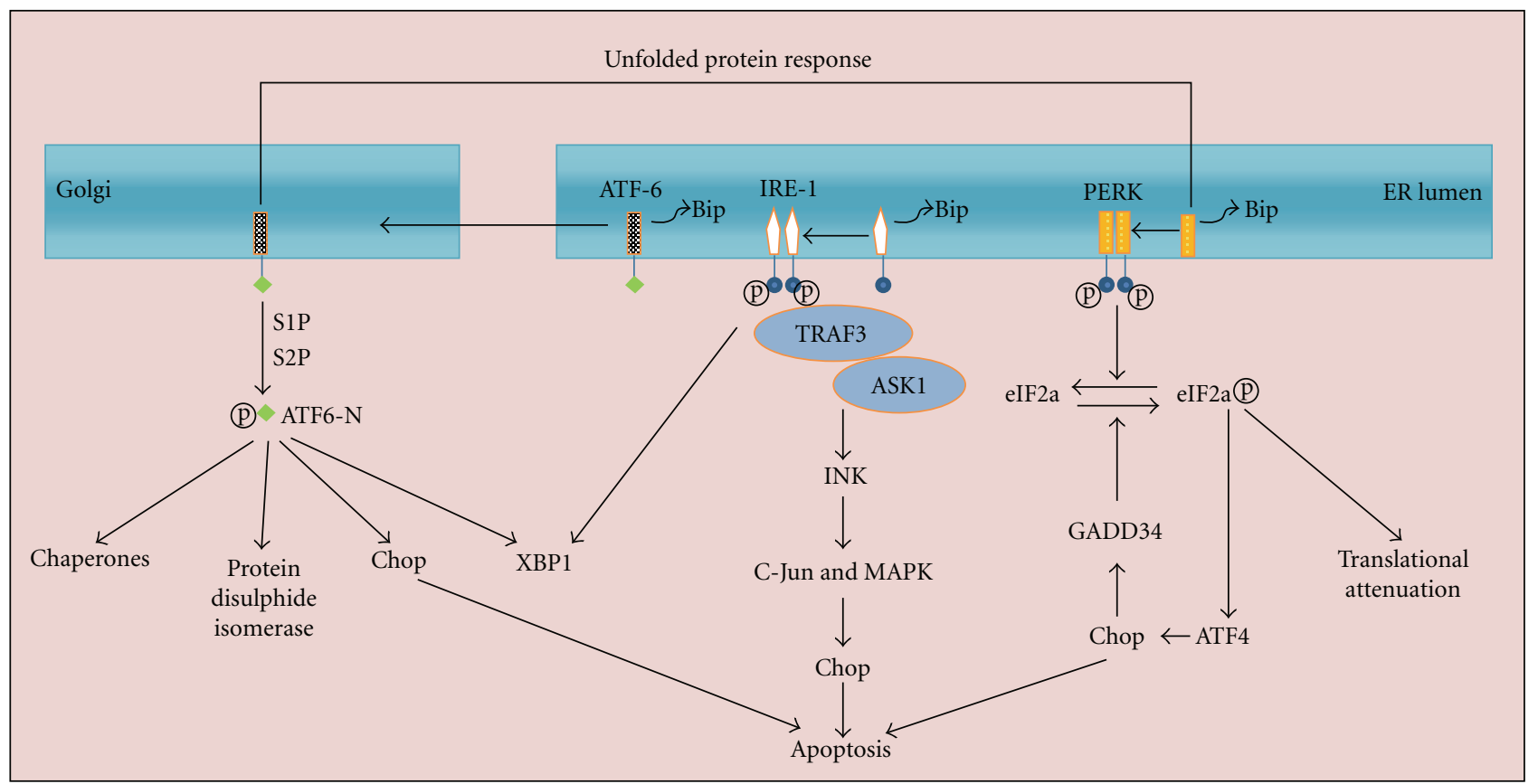

FIGURE 2: In response to ER stress, Bip separates from the three transmembrane mediators, causing the unfolded protein response to be activated. Unbound PERK then phosphorylates eIF2 $\alpha$, leading both to inhibition of new protein translation and to induction of the ATF4 transcription factor. ATF4, in turn, activates CHOP gene expression, which then promotes apoptosis and induces the growth arrest and DNA damage-inducible gene 34 (GADD34). The GADD34 phosphatase dephosphorylates eIF2 $\alpha$, thereby completing a negative feedback loop. Meanwhile, the unbound IRE1 initiates splicing of the XBP-1 mRNA. Recruitment of the TNF receptor-associated factor3 (TRAF3) and apoptosis signal-regulating kinase 1 (ASK1) to IRE1 leads to activation of c-Jun amino-terminal kinase (JNK), which in turn activates c-Jun and mitogen-activated protein kinase (MAPK) and ultimately promotes CHOP activity. Unbound ATF6 is cleaved within the Golgi apparatus by the site-1 protease (S1P) and the site-2 protease (S2P) to produce an active transcription factor fragment known as ATF6-N, which in turn activates XBP1 and CHOP; in addition, transcription of ER chaperones and protein disulphide isomerase is increased.

synthesis speed, which in turn decreases protein components entering into the ER. Subsequently, expression of the ER molecular chaperones is upregulated, and the protein folding function is accelerated in an attempt for the cell to recover homeostasis. In the event that the UPR becomes too robust or prolonged [22-25], three ER stress factors are induced to facilitate quelling of the process. The pancreatic kinase(PKR-) like ER kinase (PERK), activating transcription factor 6 (ATF6) and inositol requiring enzyme 1 (IRE1) act by binding to the key ER protein immunoglobulin heavy chain protein/glucose-regulated protein, which has a molecular weight of $78 \mathrm{kDa}$ (Bip/glucose-regulated protein 78 (GRP78)) [26]. However, Bip will dissociate from PERK, ATF6 and IRE1 under conditions of accumulated unfolded or misfolded proteins inside the ER. After such dislocation, the released PERK, ATF6, and IRE1 become activated, and relevant signal transmission is initiated (Figure 2).

A variety of other factors (over 200 known to date) participate in the process of ER stress. These factors have been divided into 11 subclasses, according to their functions: unfolded protein binding, ER protein folding quality control, regulation of cholesterol metabolism, regulation of translation, endoplasmic reticulum-associated degradation (ERAD), ubiquitination, transcription factors, protein folding, protein disulfide isomerization, heat shock proteins (HSPs), and apoptosis [27].
In 2004, Roybal et al. found that activating transcription factor 4 (ATF4), which has been identified as an important factor of ER stress, is capable of directly increasing expression of the vascular endothelial growth factor (VEGF) gene [28]. Since VEGF itself plays an important role in DR, it is possible that ER stress acts through this factor to influence the pathogenesis of DR. In fact, Ikesugi et al. demonstrated that glucose deprivation conditions induced ER stress in retinal pericytes [29], supporting the notion that ER stress participates in incidence and development of DR. Oshitari et al. also found that ER stress was involved in ocular vascular abnormalities in human DR patients [30, 31]. Further experimental investigation in animal models of diabetes and oxygen-induced retinopathy (OIR), carried out by Zhang et al., demonstrated that ER stress was activated in the affected retinas and indicated that ER Stress is a potential mediator of retinal inflammation in DR [32]. The authors also demonstrated that ER stress preconditioning could protect against retinal endothelial inflammation through activation of X-box-binding protein (XBP)1-mediated UPR and inhibition of NF- $\kappa$ B activation [33].

ER stress is well known to elicit induction effects on inflammatory factors, and inflammation is believed to play a critical role in DR; thus, many researchers are currently exploring the effects of ER stress on DR via the actions of 
inflammatory factors [34]. Our laboratory also focuses on this mechanism of DR.

\section{Identifying DR-Susceptibility and DR-Protective Genes}

The bulk of research on DR hereditary factors performed to date has focused on patients with preexisting illness (i.e., diabetes), complicating the discovery of true DRsusceptibility genes. Yet, it has been clearly observed that the occurrence and severity of DR in diabetic patients varies among individuals. Some patients develop DR relatively soon after their diabetes diagnosis, while others do not develop DR for decades. In the same sense, some cases of DR are mild, while others are severe. Neither the aggressiveness nor severity of DR have been related to control of blood sugar levels or extent of loss of control, further indicating an underlying role for genetic susceptibility. Therefore, it appears that some diabetic patients have an inherent resistance to developing the DR complication or are better equipped to limit its pathogenesis.

It has been estimated that up to $20 \%$ of patients who have suffered from diabetes for 20 years remain free of the DR complication [35]. Since there are no medicines available which can effectively control DR and mitigate its progression, it can be concluded that expression of a particular gene or set of genes in these DR-resistant patients can help to protect against diabetes/glucose-related injury to retinal vessels. Even if these genes do not protect patients from DR over their entire lifetime, they may substantially delay onset or lessen severity of DR. Therefore, identification of these genes and gaining a detailed understanding of their expression patterns will likely lead to development of new therapeutic targets for genetic-based DR treatment.

On the basis upon previously published study designs for detecting genes associated with a disease state $[36,37]$, we chose a cohort of Type 2 diabetes patients $(n=59)$ with long-standing diagnosis (20 years or more). These patients were divided into two groups: not complicated by DR (normal) and complicated by DR. Gene expression analysis was performed by microarray (GeneChip human genome U133 plus 2.0; Affymetrix, Santa Clara, Calif, USA), and statistically significant differences in expression profiles were determined by comparative analysis among the two groups. Careful analysis of the enrolled patients' demographics led to exclusion of 22 patients from the normal group and 37 patients from PDR group. From the remaining patients, 20 were selected from each group, and venous blood samples were obtained for total RNA extraction. The RNAs of six patients from each group were preferentially selected to conduct gene chip detection. We found that diabetic patients without DR complication presented with 173 overexpressed genes $(P<0.05)$, compared to the PDR group [38]. These differential expression results were confirmed by quantitative PCR.

Thereafter, the functions of these 173 genes were analyzed, and it was found that 46 were related to protein degradation and structure modification. In addition, several factors known to be involved in the ER stress process were also found. Thus, we concluded that these genes that were suppressed in PDR patients may represent genes that provide a protective effect against $\mathrm{DR}$.

\section{The Role of $\mathrm{P}^{\mathrm{IPK}}{ }^{\mathrm{IPK}}$ in $\mathrm{DR}$}

One of the differentially expressed genes in our study of nonDR diabetic patients versus PRD-afflicted diabetic patients that piqued our interest was $\mathrm{P} 58^{\mathrm{IPK}}$. This gene encodes a $58 \mathrm{kDa}$ inhibitor of the interferon-induced double-stranded RNA-activated protein kinase (also known as DNAJC3) and is a member of the Hsp40 family. First characterized for its activities as an inhibitor of the key translation-mediator eukaryotic initiation factor $2 \alpha(\mathrm{eIF} 2 \alpha)$ [39], $\mathrm{P} 58^{\mathrm{IPK}}$ has since been determined to play an essential role in preventing ER stress $[40,41]$. The mechanism by which P58 ${ }^{\mathrm{IPK}}$ affects ER stress was determined to involve inhibition of PERK activation $[42,43]$, suggesting that $\mathrm{P} 58^{\mathrm{IPK}}$ acts as a key mediator of cotranslocational ER protein degradation; moreover, this process is likely to contribute to ER homeostasis in stressed cells. Mutant mouse strains with $\mathrm{P} 58^{\mathrm{IPK}}$ gene deletion displayed glucosuria, hyperglycemia, and hypoinsulinemia [44], suggesting that $\mathrm{P} 58^{\mathrm{IPK}}$ plays important roles in maintaining normal glucose levels. P58 $8^{\mathrm{IPK}}$ must enter the endoplasmic reticulum through a translocon in order to perform its protein synthesis and folding functions. Interestingly, $\mathrm{P} 58^{\mathrm{IPK}}$ can also prevent a polypeptide chain from entering the ER through the translocon, thereby reducing protein loading of the ER and protecting cells from the stress state $[45,46]$. When polypeptide chains are excluded from the ER, they are subject to degradation by the ubiquitin system. Accordingly, it has been reported that ER stress can be experimentally induced in rat pancreatic $\beta$ cells by eliminating $\mathrm{P} 58^{\mathrm{IPK}}$ as a result, the islet cells of these rats experience significant apoptosis and develop diabetes [47].

We were the first to investigate the effect of $\mathrm{P} 58^{\mathrm{IPK}}$ on DR by performing extracorporeal experiments. Human retinal capillary endothelial cells (HRCECs) were cultured in vitro and transfected with a $\mathrm{P} 58^{\mathrm{IPK}}$ overexpressing vector or $\mathrm{P} 58^{\mathrm{IPK}}$ RNA interference (RNAi) to suppress expression. As expected, $\mathrm{P} 58^{\mathrm{IPK}}$ expression was significantly increased in cells transfected with adeno-associated virus vector(rAAV2-) P58 $8^{\mathrm{IPK}}(0.63 \pm 0.02)$, as compared to those transfected with pGIPZ-P58 ${ }^{\text {IPK }}$ RNAi $(0.23 \pm 0.01)$. P5 $58^{\text {IPK }}$ expression was not different between the control transfected cells (rAAV2-GFP and pGIPZ-GFP). ER stress was induced in the transfected cells by treating with tunicamycin and changes in the expression of $\mathrm{P} 58^{\mathrm{IPK}}$ were determined, along with that of VEGF, core/emopamil-binding protein (C/EBP) homologous protein (CHOP), ATF4, and GRP78. Apoptosis levels were also determined for the $\mathrm{P} 58^{\mathrm{IPK}}$ overexpressing cells and suppressed cells. ER stress had no effect on gene expression in cells overexpressing $\mathrm{P} 58^{\mathrm{IPK}}$, as evidenced by no difference in expression levels of ATF-4, GRP78, CHOP, and VEGF as compared to those in unstressed control cells. However, the inhibitory effect of $\mathrm{P} 58^{\mathrm{IPK}}$ on the expression 


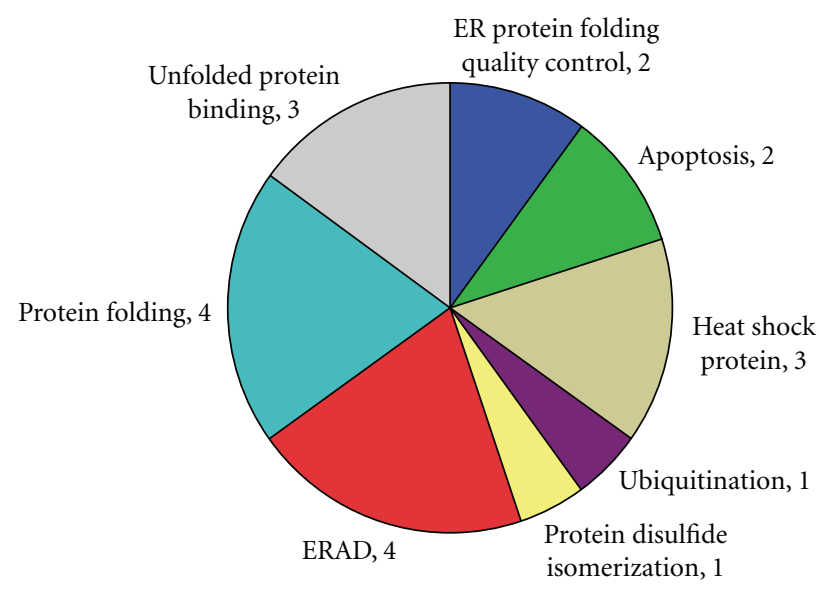

(a)

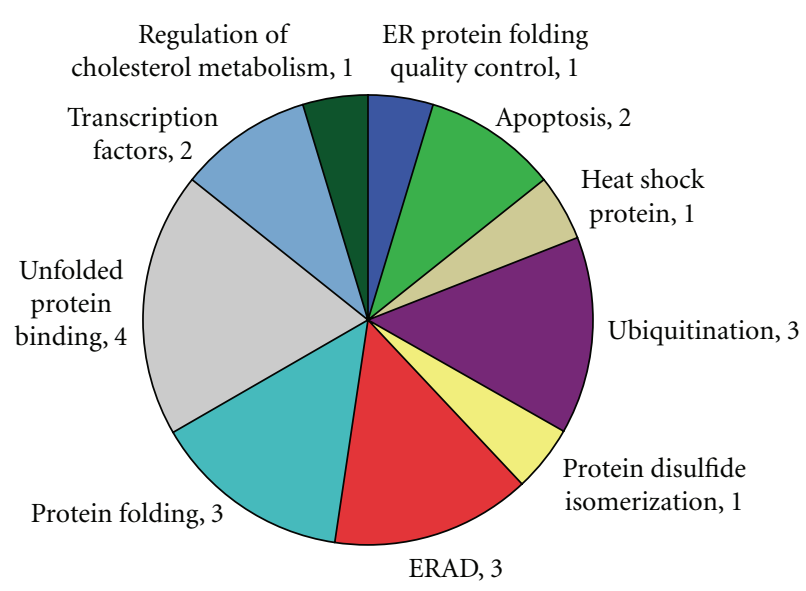

(b)

FIGURE 3: Associated functions of ER stress-related factors differentially expressed in diabetic retina in the first (a) and third (b) month after development of diabetes (numbers of proteins identified for each function are indicated). Expression levels were determined by quantitative real-time RNA polymerase chain reaction microarrays and compared to those of normal nondiabetic rats; differential expression was designated if a gene was detected at 2-fold lower levels.

of ER stress-related factors was suppressed in cells transfected with pGIPZ- P58 $8^{\mathrm{IPK}}$ RNAi. Apoptosis was also found to be significantly increased in cells transfected with pGIPZ-P58 $8^{\mathrm{IPK}}$ RNAi and not in those transfected with rAAV2-P58 ${ }^{\text {IPK }}$ [48].

We also investigated the effects of $\mathrm{P} 58^{\mathrm{IPK}}$ overexpression on the retinas of rats with sustained high glucose. A rat diabetic model was established by intraperitoneal injection of streptozotocin. Overexpression of P58 ${ }^{\mathrm{IPK}}$ was achieved by intravitreal injection of purified recombinant $\mathrm{rAAV} 2-\mathrm{P} 58^{\mathrm{IPK}}$ or transfection into cultured rat retinal capillary endothelial cells. Retinal vascular permeability was determined by assessing the Evans Blue retinal leakage. To downregulate the $\mathrm{P} 58^{\mathrm{IPK}}$ level in cultured rat retinal capillary endothelial cells, pGIPZP58 ${ }^{\text {IPK }}$ RNAi was introduced in these cells. Real-time reverse transcription- (RT-) PCR and Western blot analyses were performed to evaluate the mRNA and protein levels, respectively, of CHOP, VEGF, and tumor necrosis factor- $\alpha$ $(\mathrm{TNF}-\alpha)$. Results showed that retinal blood vessel leakage was significantly decreased in diabetic rats overexpressing $\mathrm{P} 58^{\mathrm{IPK}}$, as compared with the control diabetic rats. Both mRNA and protein levels of CHOP, TNF- $\alpha$, and VEGF were remarkably reduced in the retinas of diabetic rats overexpressing $\mathrm{P} 58^{\mathrm{IPK}}$. In vitro study further demonstrated that overexpression of $\mathrm{P} 58^{\mathrm{IPK}}$ led to the downregulation of CHOP, TNF- $\alpha$, and VEGF gene expression under high glucose conditions, whereas RNAi suppression of P58 IPK enhanced the expression of CHOP, TNF- $\alpha$, and VEGF [49].

Collectively, these studies indicated that $\mathrm{P} 58^{\mathrm{IPK}}$ functions include protecting the integrity of retinal vessels and resisting development and progression of DR. Its key role as a stabilizing endoplasmic reticulum factor led us to presume that $\mathrm{P} 58^{\mathrm{IPK}}$ contributes to $\mathrm{DR}$ by reducing incidence of ER stress through maintaining stability of the ER; these studies are underway. Nonetheless, $\mathrm{P} 58^{\mathrm{IPK}}$ appears to be a particularly promising genetic target to develop therapy to protect against DR.

\section{Which ER Stress-Related Factors Protect against DR?}

Research by our group and others are continuing to investigate the contribution of the full panel of ER stress-related factors to DR. We established a high glucose rat model in order to observe the expression changes of these ER stressrelated factors. At the same time, Ikesugi and colleagues and Roybal and colleagues $[28,29]$ reported that in the early stages of high glucose in rats the expressions of VEGF and CHOP were upregulated, but the expressions of GRP78 and ATF4 remained stable. Again, the animal experiment results were consistent with those from the extracorporeal system [50]. Therefore, the studies to determine exactly which ER stress factors play critical roles in resisting DR and the mechanisms by which they act are of clinical interest.

It is ultimately necessary to gain a comprehensive understanding of the effects of ER stress-related factors on DR. To this end, we selected 89 factors from the entire panel of known ER stress-related factors representing each of the 11 subclasses of function in ER stress, [27] and on the basis of relevant studies of ER stress from the literature [28, 32, 51-69]. Custom-made real-time PCR chips based on the rat sequences for these 89 genes were designed (SABiosciences, Gaithersburg, Md, USA) and employed for accurate detection of temporal expression changes of these factors in the retina of high glucose rats. The results indicated that 13 genes, including the $\mathrm{P} 58^{\mathrm{IPK}}$ gene, were significantly down-regulated in the high glucose rat model at 1 month old. In three-month-old high glucose rats, 12 genes were downregulated (Figure 3). In addition, we found that three key signaling pathways of ER stress (PERK, IRE1 and ATF6) were not activated in the early stage of high glucose in these rats. This phenomenon is consistent with our earlier research findings [50]. According to the results of our gene chip studies, the ERAD pathway-related 
factors were of particular interest. The effects of P58 $8^{\mathrm{IPK}}$ and ERAD in ER stress were discussed in this paper. Firstly, both have effects on maintaining functional balance of the endoplasmic reticulum and in preventing ER stress. $\mathrm{P} 58^{\mathrm{IPK}}$ mediates the transport of new unfolded proteins entering into the endoplasmic reticulum [45], while ERAD degrades the unfolded proteins that have accumulated in the endoplasmic reticulum. Removal (processing) of unfolded proteins from the endoplasmic reticulum is crucial to prevent ER stress, and is a more direct control mechanism than the transport modulation by $\mathrm{P} 58^{\mathrm{IPK}}$. Therefore, we have theorized that overexpression of ERAD-related factors in the retina might be able to boost the ERAD signaling pathways to a more robust level and prevent ER stress under high glucose conditions and halt or slow down the progress of DR.

\section{Perspectives for Future Studies}

Much like the information gleaned from studies to identify disease-susceptibility genes, data on disease-protective genes provide the foundational knowledge by which our understanding of human health is advanced and effective healthcare strategies are developed. If the pathological mechanism of DR is the proverbial "black box", then defining the gene expression profile of DR will represent a window through which we may observe and assess the situations inside of it. Using this idea as a guide to our own studies, we have gained significant insights into which ER stress-related factors participate in the onset and pathogenic process of DR and identified a promising target for DR treatment.

However, like most disease processes, DR involves many signaling pathways and physiological factors. Cross-talk and functional interactions among these factors certainly form a complex and dynamic network structure. Each individual gene that is characterized as having a protective role against DR effectively represents a single node in that entire network. Therefore, the collective analysis of the DR gene expression profile must be continued and augmented with studies to understand the influence of clinical (comorbidities) and nonphysiologic aspects (environmental factors).

\section{Acknowledgments}

This research was supported by the National Nature Science Foundation (Grant nos. 30872823 and 30801260) of the People's Republic of China and by the Tongji Hospital Foundation of New Technologies (Grant no. 2010021).

\section{References}

[1] F. H. Wang, Y. B. Liang, X. Y. Peng et al., "Risk factors for diabetic retinopathy in a rural Chinese population with type 2 diabetes: the Handan Eye Study," Acta Ophthalmologica, vol. 89, no. 4, pp. e336-e343, 2011.

[2] F. Semeraro, G. Parrinello, A. Cancarini et al., "Predicting the risk of diabetic retinopathy in type 2 diabetic patients," Journal of Diabetes and Its Complications, vol. 25, no. 5, pp. 292-297, 2011.

[3] W. Duckworth, C. Abraira, T. Moritz et al., "Glucose control and vascular complications in veterans with type 2 diabetes,"
New England Journal of Medicine, vol. 360, no. 2, pp. 129-139, 2009.

[4] X. Zhang, J. B. Saaddine, C. F. Chou et al., "Prevalence of diabetic retinopathy in the United States, 2005-2008," Journal of the American Medical Association, vol. 304, no. 6, pp. 649656, 2010.

[5] A. K. Cheung, M. K. Fung, A. C. Lo et al., "Aldose reductase deficiency prevents diabetes-induced blood-retinal barrier breakdown, apoptosis, and glial reactivation in the retina of db/db mice," Diabetes, vol. 54, no. 11, pp. 3119-3125, 2005.

[6] K. Naruse, J. Nakamura, Y. Hamada et al., "Aldose reductase inhibition prevents glucose-induced apoptosis in cultured bovine retinal microvascular pericytes," Experimental Eye Research, vol. 71, no. 3, pp. 309-315, 2000.

[7] X. Xu, Q. Zhu, X. Xia, S. Zhang, Q. Gu, and D. Luo, "Blood-retinal barrier breakdown induced by activation of protein kinase $\mathrm{C}$ via vascular endothelial growth factor in streptozotocin-induced diabetic rats," Current Eye Research, vol. 28, no. 4, pp. 251-256, 2004.

[8] P. Geraldes, J. Hiraoka-Yamamoto, M. Matsumoto et al., "Activation of PKC-and SHP-1 by hyperglycemia causes vascular cell apoptosis and diabetic retinopathy," Nature Medicine, vol. 15, no. 11, pp. 1298-1306, 2009.

[9] F. Giacco and M. Brownlee, "Oxidative stress and diabetic complications," Circulation Research, vol. 107, no. 9, pp. 10581070, 2010.

[10] A. W. Stitt, "AGEs and diabetic retinopathy," Investigative Ophthalmology \& Visual Science, vol. 51, no. 10, pp. 48674874, 2010.

[11] A. Praidou, S. Androudi, P. Brazitikos et al., "Angiogenic growth factors and their inhibitors in diabetic retinopathy," Current Diabetes Reviews, vol. 6, no. 5, pp. 304-312, 2010.

[12] N. Cheung, P. Mitchell, and T. Y. Wong, "Diabetic retinopathy," The Lancet, vol. 376, no. 9735, pp. 124-136, 2010.

[13] H. P. Hammes, Y. Feng, F. Pfister, and M. Brownlee, "Diabetic retinopathy: targeting vasoregression," Diabetes, vol. 60 , no. 1 , pp. 9-16, 2011.

[14] V. Kakkassery, S. Winterhalter, and A. M. Joussen, "Anti-VEGF inhibitors and their role in the treatment of diabetic macular oedema," Klinische Monatsblatter fur Augenheilkunde, vol. 227, no. 9, pp. 701-711, 2010.

[15] O. I. Sarygina, V. V. Neroev, and O. A. Levkina, "Role of vascular endothelial growth factor in the pathogenesis of diabetic retinopathy," Vestnik Oftalmologii, vol. 125, no. 2, pp. 58-60, 2009.

[16] R. A. Mahdy and W. M. Nada, "Evaluation of the role of vascular endothelial growth factor in diabetic retinopathy," Ophthalmic Research, vol. 45, no. 2, pp. 87-91, 2011.

[17] J. Qian, Q. Lu, Y. Tao, and Y. R. Jiang, "Vitreous and plasma concentrations of apelin and vascular endothelial growth factor after intravitreal bevacizumab in eyes with proliferative diabetic retinopahty," Retina, vol. 31, no. 1, pp. 161-168, 2011.

[18] J. F. Arevalo and R. A. Garcia-Amaris, "Intravitreal Bevacizumab for diabetic retinopathy," Current Diabetes Reviews, vol. 5, no. 1, pp. 39-46, 2009.

[19] A. Doria, "Genetics of diabetes complications," Current Diabetes Reports, vol. 10, no. 6, pp. 467-475, 2010.

[20] S. Abhary, K. P. Burdon, K. J. Laurie et al., "Aldose reductase gene polymorphisms and diabetic retinopathy susceptibility," Diabetes Care, vol. 33, no. 8, pp. 1834-1836, 2010.

[21] M. Buraczynska, P. Ksiazek, I. Baranowicz-Gaszczyk, and L. Jozwiak, "Association of the VEGF gene polymorphism with diabetic retinopathy in type 2 diabetes patients," Nephrology Dialysis Transplantation, vol. 22, no. 3, pp. 827-832, 2007. 
[22] G. Liew, R. Klein, and T. Y. Wong, "The role of genetics in susceptibility to diabetic retinopathy," International Ophthalmology Clinics, vol. 49, no. 2, pp. 35-52, 2009.

[23] J. Faitova, D. Krekac, R. Hrstka, and B. Vojtesek, "Endoplasmic reticulum stress and apoptosis," Cellular and Molecular Biology Letters, vol. 11, no. 4, pp. 488-505, 2006.

[24] A. Kapoor and A. J. Sanyal, "Endoplasmic reticulum stress and the unfolded protein response," Clinics in Liver Disease, vol. 13, no. 4, pp. 581-590, 2009.

[25] R. T. Weston and H. Puthalakath, "Endoplasmic reticulum stress and BCL-2 family members," Advances in Experimental Medicine and Biology, vol. 687, pp. 65-77, 2010.

[26] P. I. Merksamer and F. R. Papa, "The UPR and cell fate at a glance," Journal of Cell Science, vol. 123, no. 7, pp. 1003-1006, 2010.

[27] A. Bertolotti, Y. Zhang, L. M. Hendershot, H. P. Harding, and D. Ron, "Dynamic interaction of BiP and ER stress transducers in the unfolded-protein response," Nature Cell Biology, vol. 2, no. 6, pp. 326-332, 2000.

[28] C. N. Roybal, S. Yang, C. W. Sun et al., "Homocysteine increases the expression of vascular endothelial growth factor by a mechanism involving endoplasmic reticulum stress and transcription factor ATF4," Journal of Biological Chemistry, vol. 279, no. 15, pp. 14844-14852, 2004.

[29] K. Ikesugi, M. L. Mulhern, C. J. Madson et al., "Induction of endoplasmic reticulum stress in retinal pericytes by glucose deprivation," Current Eye Research, vol. 31, no. 11, pp. 947953, 2006

[30] T. Oshitari, N. Hata, and S. Yamamoto, "Endoplasmic reticulum stress and diabetic retinopathy," Vascular Health and Risk Management, vol. 4, no. 1, pp. 115-122, 2008.

[31] J. Li, J. J. Wang, Q. Yu, M. Wang, and S. X. Zhang, "Endoplasmic reticulum stress is implicated in retinal inflammation and diabetic retinopathy," FEBS Letters, vol. 583, no. 9, pp. 15211527, 2009.

[32] J. Li, J. J. Wang, and S. X. Zhang, "Preconditioning with endoplasmic reticulum stress mitigates retinal endothelial inflammation via activation of X-box binding protein 1," Journal of Biological Chemistry, vol. 286, no. 6, pp. 4912-4921, 2011.

[33] F. S. Collins, E. D. Green, A. E Guttmacher et al., "A vision for the future of genomics research," Nature, vol. 422, pp. 835$847,2003$.

[34] R. N. Frank, "Diabetic Retinopathy," New England Journal of Medicine, vol. 350, no. 1, pp. 48-58, 2004.

[35] P. J. Valk, R. G. Verhaak, M. A. Beijen et al., "Prognostically useful gene-expression profiles in acute myeloid leukemia," New England Journal of Medicine, vol. 350, no. 16, pp. 1617$1628,2004$.

[36] J. E. Gunton, R. N. Kulkarni, S. Yim et al., "Loss of ARNT/HIF1 $\beta$ mediates altered gene expression and pancreatic-islet dysfunction in human type 2 diabetes," Cell, vol. 122, no. 3, pp. 337-349, 2005.

[37] B. Li, H.-Q. Zhang, Y. Shi et al., "Overexpression of nuclear transport factor 2 may protect against diabetic retinopathy," Molecular Vision, vol. 15, pp. 861-869, 2009.

[38] G. N. Barber, S. Thompson, T. G. Lee et al., "The 58-kilodalton inhibitor of the interferon-induced double-stranded RNAactivated protein kinase is a tetratricopeptide repeat protein with oncogenic properties," Proceedings of the National Academy of Sciences of the United States of America, vol. 91, no. 10, pp. 4278-4282, 1994.

[39] D. T. Rutkowski, S. W. Kang, A. G. Goodman et al., "The role of p58IPK in protecting the stressed endoplasmic reticulum,"
Molecular Biology of the Cell, vol. 18, no. 9, pp. 3681-3691, 2007.

[40] A. G. Goodman, J. A. Smith, S. Balachandran et al., "The cellular protein P58IPK regulates influenza virus mRNA translation and replication through a PKR-mediated mechanism," Journal of Virology, vol. 81, no. 5, pp. 2221-2230, 2007.

[41] W. Yan, C. L. Frank, M. J. Korth et al., "Control of PERK elF $2 \alpha$ kinase activity by the endoplasmic reticulum stress-induced molecular chaperone P58IPK," Proceedings of the National Academy of Sciences of the United States of America, vol. 99, no. 25, pp. 15920-15925, 2002.

[42] W. C. Ladiges, S. E. Knoblaugh, J. F. Morton et al., "Pancreatic $\beta$-cell failure and diabetes in mice with a deletion mutation of the endoplasmic reticulum molecular chaperone gene P58IPK," Diabetes, vol. 54, no. 4, pp. 1074-1081, 2005.

[43] S. Oyadomari, C. Yun, E. A. Fisher et al., "Cotranslocational degradation protects the stressed endoplasmic reticulum from protein overload," Cell, vol. 126, no. 4, pp. 727-739, 2006.

[44] D. T. Rutkowski, S. W. Kang, A. G. Goodman et al., "The role of p58IPK in protecting the stressed endoplasmic reticulum," Molecular Biology of the Cell, vol. 18, no. 9, pp. 3681-3691, 2007.

[45] W. C. Ladiges, S. E. Knoblaugh, J. F. Morton et al., "Pancreatic $\beta$-cell failure and diabetes in mice with a deletion mutation of the endoplasmic reticulum molecular chaperone gene P58IPK," Diabetes, vol. 54, no. 4, pp. 1074-1081, 2005.

[46] B. Li, D. Li, G.-G. Li, H.-W. Wang, and A.-X. Yu, "P58IPK inhibition of endoplasmic reticulum stress in human retinal capillary endothelial cells in vitro," Molecular Vision, vol. 14, pp. 1122-1128, 2008.

[47] H. Yang, R. Liu, Z. Cui et al., "Functional characterization of 58-kilodalton inhibitor of protein kinase in protecting against diabetic retinopathy via the endoplasmic reticulum stress pathway," Molecular Vision, vol. 17, pp. 78-84, 2011.

[48] B. Li, H. S. Wang, G. G. Li, M. J. Zhao, and M. H. Zhao, "The role of endoplasmic reticulum stress in the early stage of diabetic retinopathy," Acta Diabetologica, vol. 48, no. 2, pp. 103-111, 2011.

[49] S. E. Thomas, L. E. Dalton, M. L. Daly, E. Malzer, and S. J. Marciniak, "Diabetes as a disease of endoplasmic reticulum stress," Diabetes/Metabolism Research and Reviews, vol. 26, no. 8, pp. 611-621, 2010.

[50] A. Salminen, A. Kauppinen, J. M. T. Hyttinen, E. Toropainen, and K. Kaarniranta, "Endoplasmic reticulum stress in agerelated macular degeneration: trigger for neovascularization," Molecular Medicine, vol. 16, no. 11-12, pp. 535-542, 2010.

[51] R. S. Hegde and H. L. Ploegh, "Quality and quantity control at the endoplasmic reticulum," Current Opinion in Cell Biology, vol. 22, no. 4, pp. 437-446, 2010.

[52] T. Hosoi and K. Ozawa, "Endoplasmic reticulum stress in disease: mechanisms and therapeutic opportunities," Clinical Science, vol. 118, no. 1, pp. 19-29, 2010.

[53] A. Kapoor and A. J. Sanyal, "Endoplasmic reticulum stress and the unfolded protein response," Clinics in Liver Disease, vol. 13, no. 4, pp. 581-590, 2009.

[54] R. P. Boot-Handford and M. D. Briggs, "The unfolded protein response and its relevance to connective tissue diseases," Cell and Tissue Research, vol. 339, no. 1, pp. 197-211, 2010.

[55] K. Kohno, "Stress-sensing mechanisms in the unfolded protein response: similarities and differences between yeast and mammals," Journal of Biochemistry, vol. 147, no. 1, pp. 27-33, 2010. 
[56] G. S. Hotamisligil, "Endoplasmic reticulum stress and the inflammatory basis of metabolic disease," Cell, vol. 140, no. 6, pp. 900-917, 2010.

[57] C. S. McAlpine, A. J. Bowes, and G. H. Werstuck, "Diabetes, hyperglycemia and accelerated atherosclerosis: evidence supporting a role for endoplasmic reticulum (ER) stress signaling," Cardiovasc Hematol Disord Drug Targets, vol. 10, no. 2, pp. 151-157, 2010.

[58] M. Kitamura, "Control of NF- $\kappa \mathrm{B}$ and inflammation by the unfolded protein response," International Reviews of Immunology, vol. 30, no. 1, pp. 4-15, 2011.

[59] C. M. Ribeiro and R. C. Boucher, "Role of endoplasmic reticulum stress in cystic fibrosis-related airway inflammatory responses," Proceedings of the American Thoracic Society, vol. 7, no. 6, pp. 387-394, 2010.

[60] A. Kaser and R. S. Blumberg, "Endoplasmic reticulum stress and intestinal inflammation," Mucosal Immunology, vol. 3, no. 1, pp. 11-16, 2010.

[61] G. S. Hotamisligil, "Inflammation and endoplasmic reticulum stress in obesity and diabetes," International Journal of Obesity, vol. 32, supplement 7, pp. S52-S54, 2008.

[62] H. Yoshida, "ER stress and diseases," FEBS Journal, vol. 274, no. 3, pp. 630-658, 2007.

[63] L. Niederreiter and A. Kaser, "Endoplasmic reticulum stress and inflammatory bowel disease," Acta Gastro-Enterologica Belgica, vol. 74, no. 2, pp. 330-333, 2011.

[64] P. C. Evans, "Regulation of pro-inflammatory signalling networks by ubiquitin: identification of novel targets for antiinflammatory drugs," Expert Reviews in Molecular Medicine, vol. 7, no. 12, pp. 1-19, 2005.

[65] T. Iwawaki, R. Akai, S. Yamanaka, and K. Kohno, "Function of IRE1 alpha in the placenta is essential for placental development and embryonic viability," Proceedings of the National Academy of Sciences of the United States of America, vol. 106, no. 39, pp. 16657-16662, 2009.

[66] J. C. Yang, C. F. Teng, H. C. Wu et al., "Enhanced expression of vascular endothelial growth factor-A in ground glass hepatocytes and its implication in hepatitis B virus hepatocarcinogenesis," Hepatology, vol. 49, no. 6, pp. 1962-1971, 2009.

[67] Y. Koyama, S. Matsuzaki, F. Gomi et al., "Induction of amyloid $\beta$ accumulation by ER calcium disruption and resultant upregulation of angiogenic factors in ARPE19 cells," Investigative Ophthalmology and Visual Science, vol. 49, no. 6, pp. 2376-2383, 2008.

[68] Z. Pawlowska, P. Baranska, H. Jerczynska, W. Koziolkiewicz, and C. S. Cierniewski, "Heat shock proteins and other components of cellular machinery for protein are up-regulated in vascular endothelial cell growth factor-activated human endothelial cells," Proteomics, vol. 5, no. 5, pp. 1217-1227, 2005.

[69] J. Li, J. J. Wang, Q. Yu, M. Wang, and S. X. Zhang, "Endoplasmic reticulum stress is implicated in retinal inflammation and diabetic retinopathy," FEBS Letters, vol. 583, no. 9, pp. 15211527, 2009. 


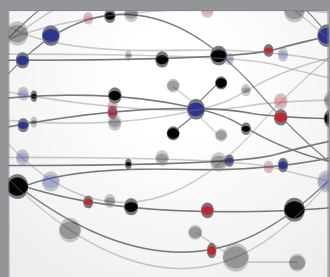

The Scientific World Journal
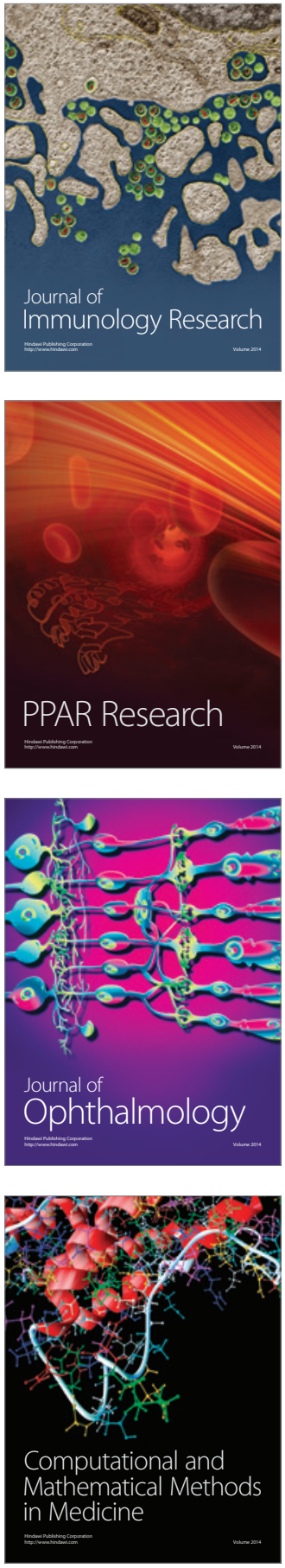

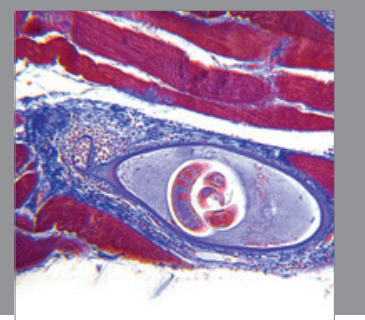

Gastroenterology

Research and Practice
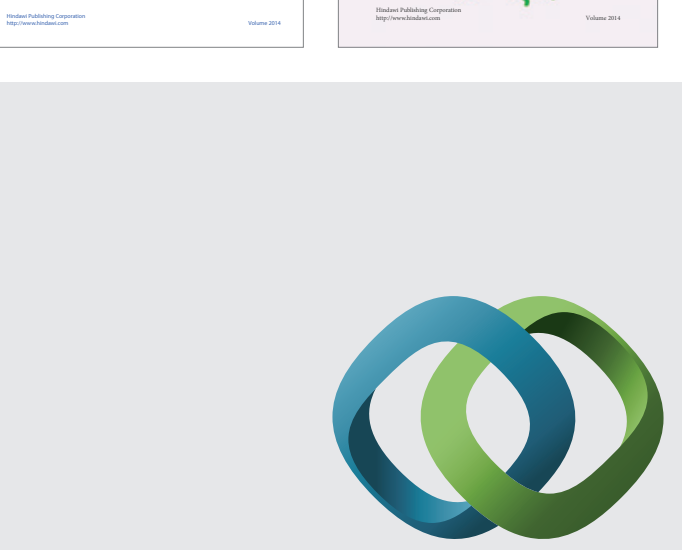

\section{Hindawi}

Submit your manuscripts at

http://www.hindawi.com
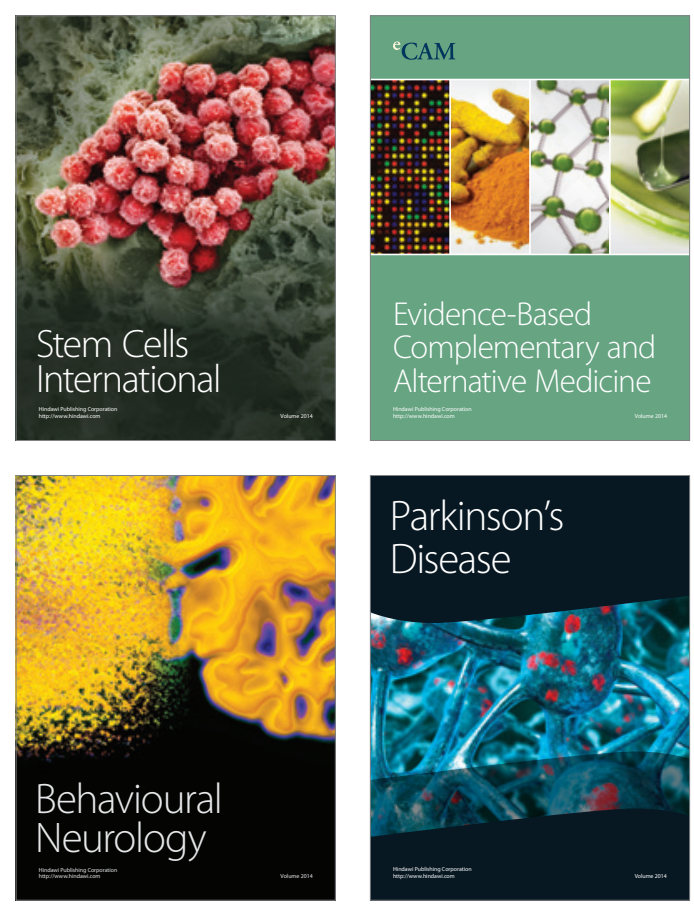

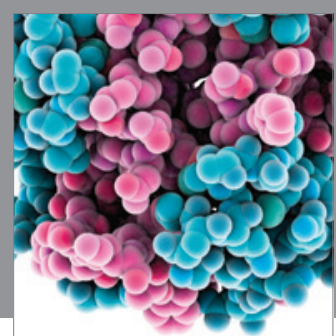

Journal of
Diabetes Research

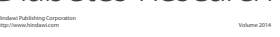

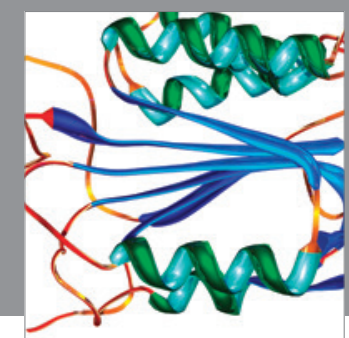

Disease Markers
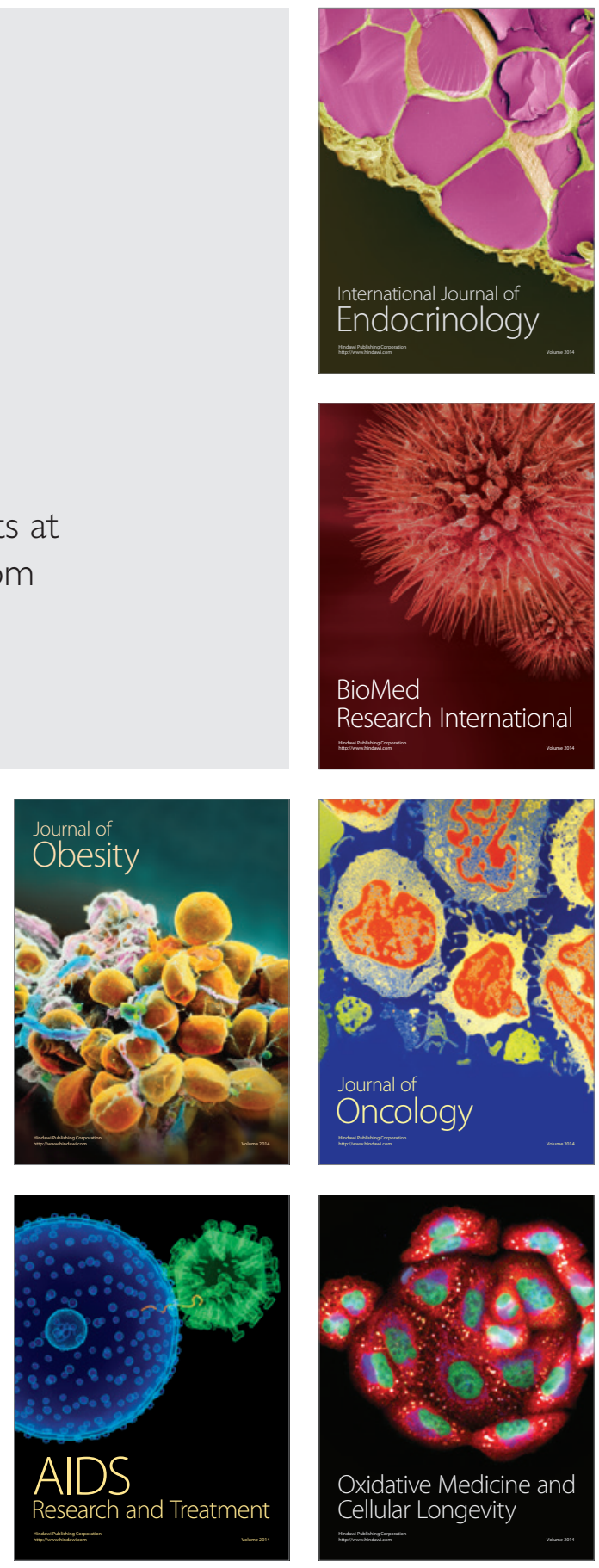